\title{
Action of sodium aurothiomalate on erythrocyte membrane
}

\author{
J M Campbell, J Reglinski, W E Smith, D Porter, R D Sturrock
}

\begin{abstract}
The number of sulphydryl groups on the erythrocyte membrane has been assessed as a function of nutritional status for two groups of patients, one receiving non-steroidal antiinflammatory drugs (NSAIDs) and the other receiving sodium aurothiomalate (Myocrisin). The patients receiving NSAIDs had a significantly higher number of sulphydryl groups in both the glucose depleted and glucose activated states than the patients receiving sodium aurothiomalate. The study focuses on the hexose transport protein where there is a specific binding site for gold using the two sulphydryl residues on helices 11 and 12 of the protein. The data suggest that the strong binding of gold to the erythrocyte membrane occurs via thiol pairs rather than by isolated sulphydryl groups and that there are possibly two further binding sites for gold on the membrane, the identities of which are still unclear.
\end{abstract}

(Ann Rheum Dis 1992; 51: 969-971)

Ellman's reagent (5,5'-dithiobis-2-nitrobenzoic acid) exchanges with sulphydryl groups and can be used to determine the number of sulphydryl groups on the exofacial surface of intact erythrocytes. 12 Using this method it was shown that there is a significant difference between the number of sulphydryl groups on erythrocytes when they are incubated in the presence and absence of glucose. ${ }^{1}$ The difference is consistent with the exposure of two sulphydryl functional groups to the plasma domain by the hexose transport protein when it binds the glucose. A comparison of erythrocytes obtained from patients with rheumatoid arthritis with those from normal healthy volunteers showed that the number of membrane sulphydryl groups was lower in the disease state. This is also true for plasma thiol groups. ${ }^{2}$ This suggested that, as in plasma, oxidation of the membrane sulphydryl groups occurs during the disease process. There is no correlation between the plasma and membrane sulphydryl concentrations, however, and consequently the redox balance of the membrane and plasma do not seem to be directly linked. ${ }^{2}$

By taking glucose depleted erythrocytes, incubating them with glucose, and monitoring the number of sulphydryl groups present with time it is possible to monitor the conformational change which exposes the two thiol sites on helices 11 and 12 of the hexose transport protein during glucose transport. $^{3}$ A significant difference in the time taken for this confor- mational change to occur is observed between erythrocytes obtained from normal healthy volunteers (50 minutes) and erythrocytes obtained from patients with rheumatoid arthritis (80 minutes). The latter were not treated with any second line or cytotoxic drugs.

Sodium aurothiomalate (Myocrisin) is one of the few drugs which is active in rheumatoid arthritis. ${ }^{4}$ Much of our in vitro work has been carried out in an attempt to understand the underlying chemistry behind this species. ${ }^{56}$ In vitro model studies of the action of sodium aurothiomalate on erythrocytes indicate that a direct interaction of the gold complex and the sulphydryl function takes place. There is also evidence to suggest that a specific thiol receptor site exists on the hexose transport protein which mediates in the cross membrane redox process associated with the interaction of sodium aurothiomalate with membrane thiol. ${ }^{57}$ These two studies suggest that there may be a link between sulphydryl status, nutritional status, and gold binding in erythrocytes and as such a clinical study has been conducted to establish whether there is a link between the in vitro ${ }^{5}$ results and the in vivo effect.

\section{Methods}

All reagents used in this study were obtained commercially unless stated otherwise. Whole blood was collected in heparinised anticoagulant tubes from 20 patients with classical or definite rheumatoid arthritis as defined by the American Rheumatism Association criteria. ${ }^{8}$ Ten of the patients were not receiving any second line or cytotoxic drugs and 10 patients were receiving sodium aurothiomalate. The patients were matched for age and sex and consisted of two groups of 10 women aged $58 \cdot 8$ (sodium aurothiomalate group) and 61.9 (group receiving non-steroidal anti-inflammatory drugs (NSAIDs)) years. The patients selected for study had been receiving sodium aurothiomalate for at least one year and were assessed to be in remission. Dosage regimens were $50 \mathrm{mg}$ weekly (one patient), every two weeks (four patients), and monthly (five patients). The disease duration of the two groups was similar ( $1-40$ years). Mean (SD) erythrocyte sedimentation rates ( $\mathrm{mm} /$ hour) were assessed as a measure of disease activity: NSAID group $65.0(16.8)$ (five patients); sodium aurothiomalate group $29 \cdot 4$ (6.6) (nine patients).

Samples were centrifuged at $1500 \mathrm{rev} / \mathrm{min}$ for 10 minutes and the supernatant and the buffy white coat were removed by suction. The resulting red cells were washed three times in 
phosphate buffered saline $(0 \cdot 125 \mathrm{M}$ sodium hydrogen phosphate; $0.154 \mathrm{M}$ sodium chloride adjusted to $\mathrm{pH} 7 \cdot 4$ with concentrated hydrochloric acid). They were then left for four hours at room temperature. At this point the glucose depleted cells ${ }^{1}$ were washed once more with phosphate buffered saline. The number of cells in a given volume of pellet was counted and 1.0 $\mathrm{ml}$ was transferred to a sterile container with $18.0 \mathrm{ml}$ of phosphate buffered saline, $1.0 \mathrm{ml}$ $0.1 \mathrm{M}$ glucose, and the container was gently mixed. At various time intervals (20 minutes) the tubes were gently mixed, a $1.5 \mathrm{ml}$ sample of the suspension withdrawn and added to a solution of Ellman's reagent $\left(1.5 \mathrm{ml}, 2 \times 10^{-4} \mathrm{M}\right)$ and the mixture allowed to react for 20 minutes. The suspensions were then centrifuged (1500 $\mathrm{rev} / \mathrm{min}$ for 10 minutes), the supernatant removed and the visible spectrum $(600-300 \mathrm{~nm})$ recorded. The concentration of Ellman's anion was calculated using Beer's law (molar absorbance $\lambda_{\max }$ for $\mathrm{ES}^{-}=13600 \mathrm{~cm}^{2} \mathrm{~mol}^{-1}, 412$ $\mathrm{nm}^{1}$ ) and used in the following equation to give the total number of thiols present on the surface of the membrane

Total number of thiols present on the erythrocyte membrane

[ES $\left.{ }^{-}\right] \times$volume $\times$Avogadro's No number of cells $\times 1000$

where $\left[\mathrm{ES}^{-}\right]$is the concentration of Ellman's anion in solution calculated using Beer's law.

The supernatants were carefully checked for lysis by eye and by spectrophotometry $(\lambda=541$ $\mathrm{nm}$ ). Lysed samples were discarded.

\section{STATISTICS}

The number of exofacial sulphydryl groups (activated and deactivated) were analysed using

\section{NSAID}

$\begin{array}{cc}\text { With } & \text { Without } \\ \text { glucose } & \text { glucose }\end{array}$

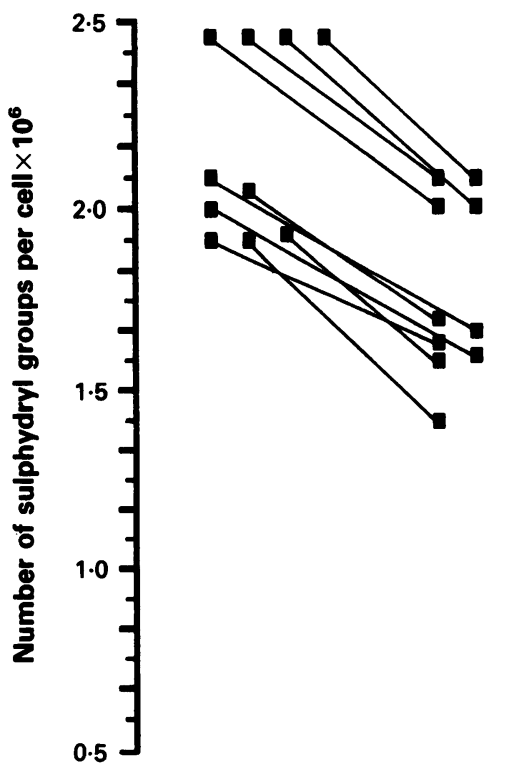

Sodium aurothiomalate

$\begin{array}{ll}\text { With } & \text { Without } \\ \text { glucose } & \text { glucose }\end{array}$

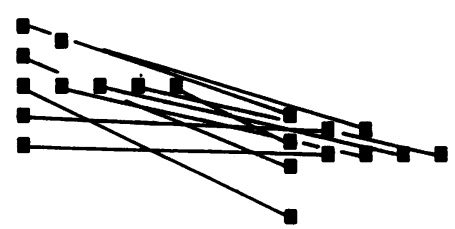

Number of sulphydryl groups in erythrocytes from 20 patients with rheumatoid arthritis, 10 receiving NSAIDs and 10 receiving sodium aurothiomalate, in the presence and absence of glucose, quoted as the number of sulphydryl functional groups for each cell. Sample pairs are linked by solid lines. the Mann-Whitney U test. The Wilcoxon matched pairs signed rank test was used to analyse the difference between the glucose activated and depleted groups.

\section{Results and discussion}

The concentration of sulphydryl groups on the erythrocyte membrane was obtained from two groups of patients with rheumatoid arthritis. One group was receiving NSAIDs and the other group sodium aurothiomalate. ${ }^{15}$ To focus on the hexose transport protein, measurements of sulphydryl status were taken at two extremes namely glucose activated and glucose depleted cells. When the number of sulphydryl groups on the cell is calculated as the number of thiols for each cell using the equation given in the preceding section the difference between the two extremes can be attributed to the exposure of the two cysteinyl residues, one on helix 11 and the other on helix 12 of the protein, when substrate is present. ${ }^{14}$

The figure shows the data for the two patient groups. As previously observed ${ }^{2}$ the number of sulphydryl groups in the patients treated with NSAIDs is higher than that of the patients treated with sodium aurothiomalate (MannWhitney $U$ test; $p<0.01$ ) in both glucose depleted and glucose activated groups (mean (range): glucose activated plus NSAID 2.28 $(2 \cdot 12-2 \cdot 74)$; glucose depleted plus NSAID $1 \cdot 82$ $(1 \cdot 72-2 \cdot 21)$; glucose activated plus sodium aurothiomalate $1.20 \quad(1 \cdot 20-1 \cdot 30)$; glucose depleted plus sodium aurothiomalate 0.98 $(0 \cdot 96-1 \cdot 05))$. In this study, however, the data are a reflection of the nutritional status of the red cells. There is a significant difference in both groups of patients between the glucose active $(p<0.01)$ and glucose depleted $(p<0.01)$ groups as determined using the Wilcoxon matched pairs signed rank test. The assay generates Ellman's anion only where there are free sulphydryl groups on the membrane. If a gold moiety is bound to the sulphydryl group the free anion is no longer detected in the assay. Therefore the observed decrease in thiol concentration is believed to result from gold bound to membrane proteins via sulphydryl residues. The change in sulphydryl concentrations as a consequence of the addition of glucose is larger for the group treated with NSAIDs $\left(4.6 \times 10^{5}\right)$ than for the group treated with sodium aurothiomalate $\left(2.2 \times 10^{5} ; \mathrm{p}<0.01\right)$. Assuming that there are roughly $3 \times 10^{5}$ copies of the hexose transport protein in each cell $^{9}{ }^{10}$ for the group treated with NSAIDs, the change represents roughly 1.5 thiols for each protein molecule. For the patients treated with sodium aurothiomalate this number decreases to 0.7 thiols for each protein, indicating that gold is penetrating the protein.

No correlation was found between these data and the clinical markers of disease duration, disease activity, or with dosages and duration of treatment with gold.

Although the change in the number of sulphydryl groups for the patients treated with sodium aurothiomalate is small, it remains significant. It is therefore possible to determine 
the time taken for the protein to change conformation. ${ }^{1}$ In this respect there is little difference between the two groups with both having about the same half life (90 minutes). This is interpreted as supporting the suggestion that the residual sulphydryl groups in the hexose transport protein of patients treated with sodium aurothiomalate is not modulated by the gold. It seems that the metal centre is not randomly distributed among the sulphydryl groups but that it binds or chelates to sulphydryl pairs within the protein. Site occupancy in the protein can be calculated on this basis to be approximately $65 \%$. This is a consequence of protein activity. The two sulphydryl residues will only be exposed during substrate binding. At this point sodium aurothiomalate may bind to the two thiolate residues. Gold interpolates into the matrix in an organised manner leaving some proteins in a non-modified form. Therefore the assay of conformational change measures changes in proteins which do not have a high gold density and are more consistent with untreated proteins - that is, what is under study in the group treated with NSAIDs.

The number of sulphydryl groups in this study is higher than that reported previously ${ }^{1}$ because the experimental protocol has been changed. A four hour incubation period was used to deplete the erythrocytes rather than the longer 16 hour period used in the initial study. This change was necessary as a result of the fragility of cells treated with sodium aurothiomalate which tended to lyse over 16 hours to an extent which interfered with the assay. The difference between the studies is evident in depleted and non-depleted cells and suggests that on removal of the cells from their natural environment, slow oxidation of the membrane sulphydryl functional groups occurs, reducing the number of effective sites with time.

The difference between the glucose activated and glucose depleted cells in the group with NSAIDs (fig) is consistent with a pair of thiol residues capable of binding one gold moiety. A comparison of the number of sulphydryl groups in the glucose depleted cells in the two patient groups suggests that gold removes a number of sulphydryl groups from the membrane which is approximately twice the difference between the glucose depleted and activated groups treated with NSAIDs - that is, one metal binding site. This assumes that there are no other factors (for example, disease severity) except the presence of gold which lower the sulphydryl content of the erythrocyte membrane in the group treated with sodium aurothiomalate with respect to the group treated with NSAIDs. This difference can be interpreted as the presence of a further two strong binding sites for gold on the membrane surface. It also confirms that gold binds to the membrane via an interaction with pairs of sulphydryl residues or with one thiol and an arrangement of other soft ligands as only this configuration creates the added stability required to prevent the metal being leached from the membrane by small molecules such as glutathione $e^{5}$ or by plasma proteins such as albumin $^{24}$ which would occur if gold was bound weakly to an isolated sulphydryl function or hard ligands (oxygen donors), or both.

Other proteins, anion transport, and certain antigen sites have a recognised number of thiol groups on the outer surface of the cell ${ }^{1}$ and could bind gold. Some of the residual thiol groups which remain active in depleted and activated erythrocytes are undoubtedly due to these proteins. This study clearly links the sulphydryl functional groups of the hexose transport protein and binding of gold. This connection could have implications for the mechanism of action of the related gold compound auranofin. The activity of this complex is usually discussed in relation to the triethylphosphine ligand making the molecule membrane permeable ${ }^{11}$ with little attention being paid to the thio-sugar ligand. This study suggests that the thio-sugar ligand may be important in the efficacy of auranofin as it may target the molecule to the hexose transport protein which could then remove and bind gold.

We thank the Arthritis and Rheumatism Council and the Nuffield Foundation for financial support for JMC and JR respectively.

1 Chilles C, Mulheron M, McCrae F, et al. Concentration and reactivity of the sulphydryl group population on the membrane of intact erythrocytes in patients with rheumatoid arthritis. Ann Rheum Dis 1990; 49: 668-71.

2 McCrae F, Reglinski J, Smith W E, Brzeski M, Sturrock $R$ D. The action of myocrisin on membrane bound sulphydryl groups. Clin Chim Acta 1990; 195: 41-6.

3 Holman G D, Rees W D. Photolabelling of the hexose transport protein at external and internal sites: fragmentation patterns and evidence for a conformational change. Biochim Biophys Acta 1987; 897: 395-405.

4 Rae K, McKay C N N, Brown D H, Smith W E, Lewis D, Capell H A. Early and late changes in sulphydryl group and copper protein concentrations and activities during drug treatment with aurothiomalate and auranofin. Ann Rheum Dis 1986; 45: 839-46.

5 Smith W E, Reglinski J, Hoey S, Brown D H, Sturrock R D. The action of sodium gold (I) thiomalate on erythrocyte membrane. Inorganic Chemistry 1990; 29: 5190-6.

6 Reglinski J, Hoey S, Smith W E. Exchange reactions between disulphides and myocrisin: an in-vitro model for a mechanism in chrysotherapy. Inorganica Chimica Acta 1988; 152: 261-4.

7 Reglinski J, Hoey S, Smith W E, Sturrock R D. Cellular response to oxidative stress at sulphydryl group receptor sites on the erythrocyte membrane. $\mathcal{F}$ Biol Chem 1988; 263: $12360-6$.

8 Arnett F C, Edworthy S M, Bloch D A, et al. The American Rheumatism Association 1987 revised criteria for the classification of rheumatoid arthritis. Arthritis Rheum 1988; 31: 315-24.

9 Lin S, Spudich J A. Biochemical studies on the mode of action of cytochalasin B. F Biol Chem 1974; 249: 5778-83.

10 Holman G A, Parker B A, Midgley P J W. Exofacial photoaffinity labelling of the human erythrocyte sugar transporter. Biochim Biophys Acta 1986; 855: 115-26.

11 Sadler P J, Berners-Price S J. Phosphine and metal phosphine complexes: relationship of chemistry to anti-cancer and other biological activity. Structure and Bonding 1988; 70: 27-102. 